\title{
Modeling and Control of a Quadrotor UAV with Tilting Propellers
}

\author{
Markus Ryll, Heinrich H. Bülthoff, and Paolo Robuffo Giordano
}

\begin{abstract}
Standard quadrotor UAVs possess a limited mobility because of their inherent underactuation, i.e., availability of 4 independent control inputs (the 4 propeller spinning velocities) vs. the 6 dofs parameterizing the quadrotor position/orientation in space. As a consequence, the quadrotor pose cannot track an arbitrary trajectory over time (e.g., it can hover on the spot only when horizontal). In this paper, we propose a novel actuation concept in which the quadrotor propellers are allowed to tilt about their axes w.r.t. the main quadrotor body. This introduces an additional set of 4 control inputs which provides full actuation to the quadrotor position/orientation. After deriving the dynamical model of the proposed quadrotor, we formally discuss its controllability properties and propose a nonlinear trajectory tracking controller based on dynamic feedback linearization techniques. The soundness of our approach is validated by means of simulation results.
\end{abstract}

\section{INTRODUCTION}

Unmanned aerial vehicles (UAVs) are a popular research field as attested by the increasing attention received over the last years, see, e.g., [1], [2], [3], [4], [5]. The possibility of using UAVs in various tasks such as data collection, search and rescue missions, or exploration and surveillance of unaccessible disaster areas are fascinating both from a scientific viewpoint and from the potential beneficial impact on our society. Recent advances in this field have also been possible thanks to the progress, over the last decade, in microelectromechanical systems and sensors (MEMS) and in the computational power of microcontrollers, which allowed to realize new micro UAVs with affordable costs.

While the small size of such UAVs is especially suitable for surveillance tasks and/or indoor navigation, several groups are also starting to address the interesting possibility to allow for an actual interaction with the environment, either in the form of direct contact [6], [7] or by considering simple grasping/manipulation tasks [8], [9]. This research is motivated by the need of transitioning from the classical topic of "autonomous flying vehicles" into the more roboticoriented "autonomous flying service robots", that is flying robots able to accomplish typical manipulation tasks [10]. However, major advancements in the complexity of the tasks envisioned for future UAVs should also be followed by innovative breakthroughs on the mechanical and actuation sides. In this respect, several possibilities have been proposed in the past literature spanning different concepts: ducted-fan

M. Ryll and P. Robuffo Giordano are with the Max Planck Institute for Biological Cybernetics, Spemannstraße 38, 72076 Tübingen, Germany \{markus.ryll,prg\}@tuebingen.mpg.de.

H. H. Bülthoff is with the Max Planck Institute for Biological Cybernetics, Spemannstraße 38, 72076 Tübingen, Germany, and with the Department of Brain and Cognitive Engineering, Korea University, Anamdong, Seongbuk-gu, Seoul, 136-713 Korea hhb@tuebingen.mpg.de. designs [11], tilt-wing mechanisms [12], [13], or tilt-rotor actuations [14], [15].

Taking inspiration from these latter works, in this paper we propose a novel actuation concept for a quadrotor UAV in which the (usually fixed) propellers are allowed to tilt about the axes connecting them to the main body frame, thus realizing a 'quad(tilt-)rotor UAV'. Indeed, one of the limitations of the classical quadrotor design lies in its inherent underactuation: presence of only 4 independent control inputs (the 4 propeller spinning velocities) does not allow to independently control the position and orientation of the quadrotor at the same time. For instance, in quasi-hover conditions, an horizontal translation necessarily implies a change in the attitude or, symmetrically, a quadrotor can hover in place only when being horizontal w.r.t. the ground plane. On the other hand, in this paper we will show that by means of these additional 4 actuated dofs it is possible to gain full controllability over the quadrotor position and orientation, thus rendering it, as a matter of fact, a fully actuated flying rigid body. Apart from the novel challenges that this design represents from a control point of view, we believe that endowing UAVs with full 6 dofs mobility will represent an important feature in many future applications, especially those involving interaction/manipulation in cluttered environments where precise force/motion control will be a mandatory need.

Goals of this paper are then: $(i)$ to derive the dynamical model of the proposed quadrotor with tilting propellers (Sect. II), (ii) to formally analyze its controllability properties and to devise a closed-loop controller able to asymptotically track an arbitrary desired trajectory for the quadrotor position/orientation in space (Sect. III), and (iii) to present simulation results assessing the effectiveness and robustness of this control strategy. Finally, Sect. V will conclude the paper and discuss future perspectives.

\section{QUADROTOR DYNAMIC MODEL}

The quadrotor analyzed in this paper can be considered as a connection of 5 main rigid bodies in relative motion among themselves: the quadrotor body itself $B$, and the 4 propeller groups $P_{i}$. These consist of the propeller arm hosting the motor responsible for the tilting actuation mechanism, and the propeller itself connected to the rotor of the motor responsible for the propeller spinning actuation (see Fig. 1). The aim of this Section is to derive the equations of motion of this multi-body system. 


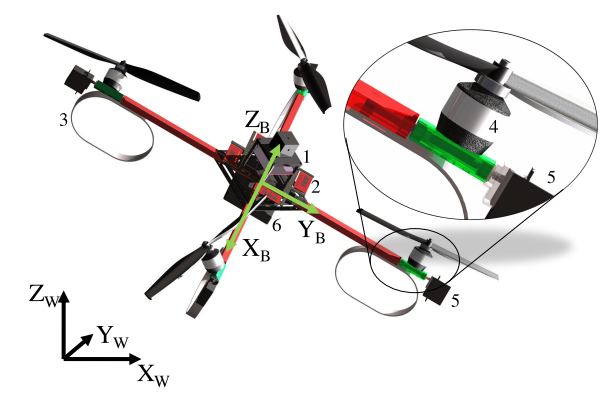

Fig. 1: CAD model of the quadrotor with tilting propellers. The model is based on a quadrotor from mikrokopter.de, and is composed of: (1) Micro controller, (2) Brushless controller, (3) Lander, (4) Propeller motor, (5) Tilting actuator, (6) Battery.

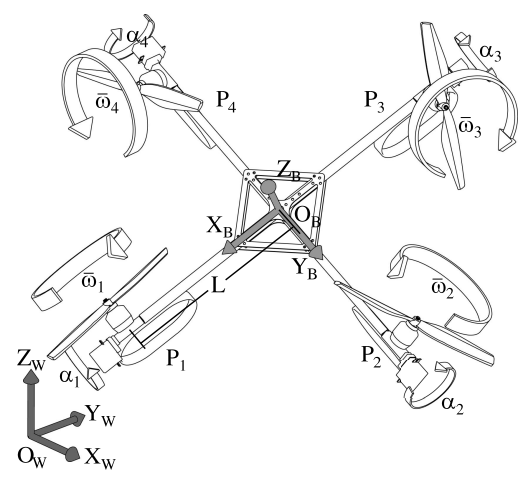

Fig. 2: Schematic view of the quadrotor considered in this paper. The overall center of mass is assumed to be in the body frame center. The symbol $L$ represents the length of all propeller arms, $\omega_{i}, i=1 \ldots 4$, the propeller rotation speed and $\alpha_{i}, i=1 \ldots 4$, the orientation of the propeller group.

\section{A. Preliminary definitions}

Let $\mathcal{F}_{W}:\left\{\boldsymbol{O}_{W} ; \boldsymbol{X}_{W}, \boldsymbol{Y}_{W}, \boldsymbol{Z}_{W}\right\}$ be a world inertial frame and $\mathcal{F}_{B}:\left\{\boldsymbol{O}_{B} ; \boldsymbol{X}_{B}, \boldsymbol{Y}_{B}, \boldsymbol{Z}_{B}\right\}$ a moving frame attached to the quadrotor body at its center of mass (see Fig. 2). We also define $\mathcal{F}_{P_{i}}:\left\{\boldsymbol{O}_{P_{i}} ; \boldsymbol{X}_{P_{i}}, \boldsymbol{Y}_{P_{i}}, \boldsymbol{Z}_{P_{i}}\right\}, i=$ $1 \ldots 4$, as the frames associated to the $i$-th propeller group, with $\boldsymbol{X}_{P_{i}}$ representing the tilting actuation axis and $\boldsymbol{Z}_{P_{i}}$ the propeller actuated spinning (thrust $T_{i}$ ) axis (see Fig. 3).

As usual, we let ${ }^{1} \boldsymbol{R}_{2} \in S O(3)$ be the rotation matrix representing the orientation of frame 2 w.r.t. frame 1: therefore, ${ }^{W} \boldsymbol{R}_{B}$ will represent the orientation of the body frame w.r.t. the world frame, while ${ }^{B} \boldsymbol{R}_{P_{i}}$ the orientation of the

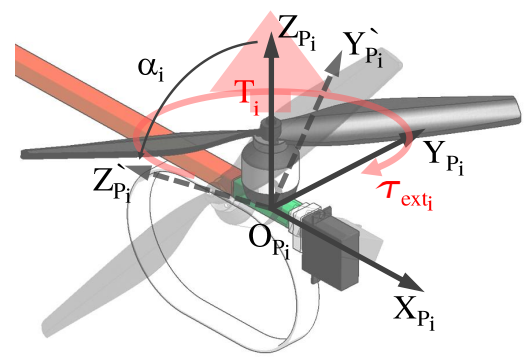

Fig. 3: $i$-th tilting arm visualizing the body frame $\mathcal{F}_{P_{i}}$, the associated propeller thrust $T_{i}$, torque $\tau_{\text {ext }}$ and the propeller tilt angle $\alpha_{i}$ propeller group $i$-th frame w.r.t. the body frame. By denoting with $\alpha_{i} \in \mathbb{R}$ the propeller tilt angle about axis $\boldsymbol{X}_{P_{i}}$, it follows from Fig. 2 that $^{1}$

$$
{ }^{B} \boldsymbol{R}_{P_{i}}=\boldsymbol{R}_{Z}\left((i-1) \frac{\pi}{2}\right) \boldsymbol{R}_{X}\left(\alpha_{i}\right), \quad i=1 \ldots 4 .
$$

Similarly, we also let

$$
{ }^{B} \boldsymbol{O}_{P_{i}}=\boldsymbol{R}_{Z}\left((i-1) \frac{\pi}{2}\right)\left[\begin{array}{l}
L \\
0 \\
0
\end{array}\right], \quad i=1 \ldots 4
$$

be the origin of the propeller frames $\mathcal{F}_{P_{i}}$ in the body frame with $L$ being the distance of $\boldsymbol{O}_{P_{i}}$ from $\boldsymbol{O}_{B}$.

Summarizing, the quadrotor configuration is completely determined by the body position $\boldsymbol{p}={ }^{W} \boldsymbol{O}_{B} \in \mathbb{R}^{3}$ and orientation ${ }^{W} \boldsymbol{R}_{B}$ in the world frame, and by the 4 tilt angles $\alpha_{i}$ specifying the propeller group orientations in the body frame (rotations about $\boldsymbol{X}_{P_{i}}$ ). We omit the propeller spinning angles about $\boldsymbol{Z}_{P_{i}}$ as configuration variables, although the propeller spinning velocities $\bar{w}_{i}$ about $\boldsymbol{Z}_{P_{i}}$ will be part of the system model (see next Sections).

\begin{tabular}{|l|l|}
\hline Symbols & Definitions \\
\hline \hline $\mathcal{F}_{W}$ & inertial world frame \\
\hline $\mathcal{F}_{B}$ & quadrotor body frame $B$ \\
\hline $\mathcal{F}_{P_{i}}$ & $i$-th propeller group frame $P_{i}$ \\
\hline $\boldsymbol{p}$ & position of $B$ in $\mathcal{F}_{W}$ \\
\hline${ }^{W} \boldsymbol{R}_{B}$ & rotation matrix from $\mathcal{F}_{B}$ to $\mathcal{F}_{W}$ \\
\hline${ }^{B} \boldsymbol{R}_{P_{i}}$ & rotation matrix from $\mathcal{F}_{P_{i}}$ to $\mathcal{F}_{B}$ \\
\hline$\alpha_{i}$ & $i$-th propeller tilt angle about $\boldsymbol{X}_{P_{i}}$ \\
\hline $\bar{\omega}_{i}$ & $i$-th propeller spinning velocity about $\boldsymbol{Z}_{P_{i}}$ \\
\hline $\boldsymbol{\omega}_{B}$ & angular velocity of $B$ in $\mathcal{F}_{B}$ \\
\hline $\boldsymbol{\tau}_{e x t_{i}}$ & $i$-th propeller air drag torque about $\boldsymbol{Z}_{P_{i}}$ \\
\hline $\boldsymbol{T}_{i}$ & $i$-th propeller thrust along $\boldsymbol{Z}_{P_{i}}$ \\
\hline$\tau_{P_{i}}$ & motor torque actuating $\boldsymbol{X}_{P_{i}}$ \\
\hline$\tau_{\bar{\omega}_{i}}$ & motor torque actuating $\boldsymbol{Z}_{P_{i}}$ \\
\hline$m$ & total mass \\
\hline $\boldsymbol{I}_{P}$ & inertia of the $i$-th propeller group $P_{i}$ \\
\hline $\boldsymbol{I}_{B}$ & inertia of the quadrotor body $B$ \\
\hline$k_{f}$ & propeller thrust coefficient \\
\hline$k_{m}$ & propeller drag coefficient \\
\hline$L$ & distance of $\mathcal{F}_{P_{i}}$ from $\mathcal{F}_{B}$ \\
\hline$g$ & gravity constant \\
\hline
\end{tabular}

\section{B. Equations of motion}

By exploiting standard techniques (e.g., Netwon-Euler procedure), it is possible to derive a complete description of the quadrotor dynamic model by considering the forces/moments generated by the propeller motion, as well as any cross-coupling due to gyroscopic and inertial effects arising from the relative motion of the 5 bodies composing the quadrotor. As for aerodynamic forces/torques, we will only consider those responsible for the quadrotor actuation and neglect any additional second-order effects/disturbances. We will now detail the main conceptual steps needed to derive the quadrotor dynamical model.

\footnotetext{
${ }^{1}$ Throughout the following, $\boldsymbol{R}_{X}(\theta), \boldsymbol{R}_{Y}(\theta), \boldsymbol{R}_{Z}(\theta)$ will denote the canonical rotation matrixes about the $\boldsymbol{X}, \boldsymbol{Y}, \boldsymbol{Z}$ axes of angle $\theta$, respectively.
} 
To this end, let $\boldsymbol{\omega}_{B} \in \mathbb{R}^{3}$ be the angular velocity of the quadrotor body $B$ expressed in the body frame ${ }^{2}$, and consider the $i$-th propeller group $P_{i}$. The angular velocity of the propeller (in its frame) is

$$
\boldsymbol{\omega}_{P_{i}}={ }^{B} \boldsymbol{R}_{P_{i}}^{T} \boldsymbol{\omega}_{B}+\left[\begin{array}{lll}
\dot{\alpha}_{i} & 0 & \bar{w}_{i}
\end{array}\right]^{T},
$$

where $\bar{w}_{i} \in \mathbb{R}$ is the spinning velocity of the propeller about $\boldsymbol{Z}_{P_{i}}$. This results in an angular acceleration

$$
\dot{\boldsymbol{\omega}}_{P_{i}}={ }^{B} \boldsymbol{R}_{P_{i}}^{T} \dot{\boldsymbol{\omega}}_{B}+{ }^{B} \dot{\boldsymbol{R}}_{P_{i}}^{T} \boldsymbol{\omega}_{B}+\left[\begin{array}{lll}
\ddot{\alpha}_{i} & 0 & \dot{\bar{w}}_{i}
\end{array}\right]^{T} .
$$

By applying the Euler equations of motion, it follows that

$$
\boldsymbol{\tau}_{P_{i}}=\boldsymbol{I}_{P_{i}} \dot{\boldsymbol{\omega}}_{P_{i}}+\boldsymbol{\omega}_{P_{i}} \times \boldsymbol{I}_{P_{i}} \boldsymbol{\omega}_{P_{i}}-\boldsymbol{\tau}_{e x t_{i}} .
$$

Here, $\boldsymbol{I}_{P_{i}} \in \mathbb{R}^{3 \times 3}$ is the (constant) symmetric and positive definite Inertia matrix of the propeller group, and $\boldsymbol{\tau}_{\text {ext }}$ any external torque applied to the propeller. As usual, see e.g. [3], we assume that there exists a counter-rotating torque about the $\boldsymbol{Z}_{P_{i}}$ axis caused by air drag and modeled as

$$
\boldsymbol{\tau}_{\text {ext }_{i}}=\left[\begin{array}{lll}
0 & 0 & -k_{m} \omega_{P_{i_{Z}}}\left|\omega_{P_{i_{Z}}}\right|
\end{array}\right]^{T}, \quad k_{m}>0 .
$$

By considering now the quadrotor body $B$ and the four propellers $P_{i}$, one obtains

$$
\boldsymbol{\tau}_{B}=\boldsymbol{I}_{B} \dot{\boldsymbol{\omega}}_{B}+\boldsymbol{\omega}_{B} \times \boldsymbol{I}_{B} \boldsymbol{\omega}_{B}+\sum_{i=1}^{4}{ }^{B} \boldsymbol{R}_{P_{i}} \boldsymbol{\tau}_{P_{i}},
$$

with $\boldsymbol{I}_{B} \in \mathbb{R}^{3 \times 3}$ being the (constant) symmetric and positive definite Inertia matrix of $B$. The (external) torques $\boldsymbol{\tau}_{B} \in \mathbb{R}^{3}$ acting on $B$ are due to the moments of the four propeller forces (thrusts) along the $\boldsymbol{Z}_{P_{i}}$ axes

$$
\boldsymbol{T}_{P_{i}}=\left[\begin{array}{lll}
0 & 0 & k_{f} \\
\bar{w}_{i} \mid \bar{w}_{i}
\end{array}\right]^{T}, \quad k_{f}>0,
$$

acting at ${ }^{B} \boldsymbol{O}_{P_{i}}$ in $\mathcal{F}_{B}$ (the propeller drag torques are already accounted for in the terms $\tau_{P_{i}}$ ). Therefore

$$
\boldsymbol{\tau}_{B}=\sum_{i=1}^{4}\left({ }^{B} \boldsymbol{O}_{P_{i}} \times{ }^{B} \boldsymbol{R}_{P_{i}} \boldsymbol{T}_{P_{i}}\right) .
$$

As for the translational dynamics, we assume for simplicity that the barycenter of each propeller group $P_{i}$ coincides with $\boldsymbol{O}_{P_{i}}$. This allows us to neglect inertial effects on the propeller groups due to the quadrotor body acceleration in space. Therefore, by recalling that $\boldsymbol{p}={ }^{W} \boldsymbol{O}_{B}$ is the quadrotor body position in world frame, one has

$$
m \ddot{\boldsymbol{p}}=m\left[\begin{array}{c}
0 \\
0 \\
-g
\end{array}\right]+{ }^{W} \boldsymbol{R}_{B} \sum_{i=1}^{4}{ }^{B} \boldsymbol{R}_{P_{i}} \boldsymbol{T}_{P_{i}}
$$

where $m$ is the total mass of the quadrotor and propeller bodies and $g$ the scalar gravity constant.

Summarizing, equations (1)-(3)-(6) describe the rotational/translational dynamics of the quadrotor body and propeller groups. Note that the inputs of this model are the motor torques actuating the propeller tilting axes $\boldsymbol{X}_{P_{i}}$ and spinning axes $\boldsymbol{Z}_{P_{i}}$. These are denoted as $\tau_{\alpha_{i}}=\boldsymbol{\tau}_{P_{i}}^{T} \boldsymbol{X}_{P_{i}} \in \mathbb{R}$

\footnotetext{
${ }^{2}$ In the following, we will assume that every quantity is expressed in its own frame, e.g., $\boldsymbol{\omega}_{B}={ }^{B} \boldsymbol{\omega}_{B}$.
}

and $\tau_{\bar{w}_{i}}=\boldsymbol{\tau}_{P_{i}}^{T} \boldsymbol{Z}_{P_{i}} \in \mathbb{R}, i=1 \ldots 4$, respectively, for a total of $4+4=8$ independent control torques (inputs). The propeller spinning velocities $\bar{w}_{i}$ (actuated by $\tau_{\bar{w}_{i}}$ ) will then generate the forces (4) and torques (2)-(5) affecting the translational/rotational motion of the quadrotor body $B$ as a function of its current configuration, in particular of the tilting angles $\alpha_{i}$ actuated by $\tau_{\alpha_{i}}$.

\section{CONTROL DESIGN}

The control problem considered in this paper is an output tracking problem: we want to track, with the available inputs, a desired (and arbitrary) trajectory $\left(\boldsymbol{p}_{d}(t), \boldsymbol{R}_{d}(t)\right) \in \mathbb{R}^{3} \times$ $S O(3)$ for the body position $\boldsymbol{p}$ and orientation ${ }^{W} \boldsymbol{R}_{B}$ taken as output functions. Note that this problem would clearly be ill-posed for a standard quadrotor with fixed propellers because of its inherent underactuation: in fact, since standard quadrotors have only 4 control inputs (usually, the 4 spinning propeller velocities $\bar{w}_{i}$ ), it is easy to prove that one can impose an arbitrary desired trajectory only to 4 independent outputs, namely, the quadrotor position $\boldsymbol{p}$ and yaw angle $\psi \in \mathbb{R}$ (the so-called quadrotor flat outputs, see, e.g., [16], [17]). The remaining 2 configuration variables specifying the quadrotor orientation cannot be freely assigned but are completely determined by the trajectory chosen for the flat outputs and its derivatives. However, as explained before, the particular actuation mechanism for the quadrotor considered in this paper allows for 8 independent control inputs, so that the stated control problem is in principle well-posed ${ }^{3}$.

The dynamic model detailed in the previous Section is useful for simulation purposes as it captures the main effects of the quadrotor motion in space (apart from any unmodeled aerodynamics forces/torques). However, some simplifications are needed in order to transform it into a model suited for control design. First, as in many practical situations, we assume that the motors actuating the tilting/spinning axes are implementing a fast high-gain local controller able to impose desired speeds $w_{\alpha_{i}}=\dot{\alpha}_{i}$ and $\bar{w}_{i}$ with negligible transients ${ }^{4}$. This allows us to neglect the motor dynamics, and to consider $w_{\alpha_{i}}$ and $\bar{w}_{i}, i=1 \ldots 4$, as (virtual) control inputs in place of the motor torques $\tau_{\alpha_{i}}$ and $\tau_{\bar{w}_{i}}$. Second, in this simplified model we also neglect the internal gyroscopic/inertial effects by considering them as second-order disturbances to be rejected by the controller. The validity of these assumptions will be thoroughly discussed in Sect. IV where the proposed controller will be tested on the complete dynamic model of Sect. II representing the actual dynamics of the quadrotor.

Let us define $\boldsymbol{\alpha}=\left[\alpha_{1} \ldots \alpha_{4}\right]^{T} \in \mathbb{R}^{4}, \boldsymbol{w}_{\alpha}=$ $\left[w_{\alpha_{1}} \ldots w_{\alpha_{4}}\right]^{T} \in \mathbb{R}^{4}$ and $\boldsymbol{w}=\left[\bar{w}_{1}\left|\bar{w}_{1}\right| \ldots \bar{w}_{4}\left|\bar{w}_{4}\right|\right]^{T} \in \mathbb{R}^{4}$. Note that elements of vector $\boldsymbol{w}$ are the signed squares of the spinning velocities $\bar{w}_{i}$, as the torques and forces in (2)-(4) are a function of these quantities. Therefore, in the following analysis, $w_{i}=\bar{w}_{i}\left|\bar{w}_{i}\right|$ will be considered as input 'spinning

\footnotetext{
${ }^{3}$ The proposed quadrotor design is actually overactuated for the chosen output function, since 8 independent control inputs are available to control the 6 independent dofs of the quadrotor body $B$.

${ }^{4}$ For instance, in the standard quadrotor case, the spinning velocities $\bar{w}_{i}$ are usually taken as control inputs.
} 
velocity' of the $i$-th propeller, with the understanding that one can always recover the actual speed $\bar{w}_{i}=\operatorname{sign}\left(w_{i}\right) \sqrt{\left|w_{i}\right|}$.

Under the stated assumptions, the quadrotor dynamic model can be simplified into

$$
\left\{\begin{aligned}
\ddot{\boldsymbol{p}} & =\left[\begin{array}{c}
0 \\
0 \\
-g
\end{array}\right]+\frac{1}{m}{ }^{W} \boldsymbol{R}_{B} \boldsymbol{F}(\boldsymbol{\alpha}) \boldsymbol{w} \\
\dot{\boldsymbol{\omega}}_{B} & =\boldsymbol{I}_{B}^{-1} \boldsymbol{\tau}(\alpha) \boldsymbol{w} \\
\dot{\boldsymbol{\alpha}} & =\boldsymbol{w}_{\alpha} \\
{ }^{W} \dot{\boldsymbol{R}}_{B} & ={ }^{W} \boldsymbol{R}_{B}\left[\boldsymbol{\omega}_{B}\right]_{\wedge}
\end{aligned}\right.
$$

with $[\cdot]_{\wedge}$ being the usual map from $\mathbb{R}^{3}$ to $s o(3)$, and

$$
\begin{gathered}
\boldsymbol{F}(\boldsymbol{\alpha})=\left[\begin{array}{cccc}
0 & -k_{f} s_{2} & 0 & k_{f} s_{4} \\
-k_{f} s_{1} & 0 & k_{f} s_{3} & 0 \\
k_{f} c_{1} & -k_{f} c_{2} & k_{f} c_{3} & -k_{f} c_{4}
\end{array}\right], \\
\boldsymbol{\tau}(\boldsymbol{\alpha})= \\
{\left[\begin{array}{ccc}
0 & -L k_{f} c_{2}-k_{m} s_{2} & 0 \\
-L k_{f} c_{1}+k_{m} s_{1} & 0 & L k_{f} c_{3}-k_{m} s_{3} \\
-L k_{f} s_{1}-k_{m} c_{1} & L k_{f} s_{2}-k_{m} c_{2} & -L k_{f} s_{3}-k_{m} c_{3} \\
L k_{f} c_{4}+k_{m} s_{4} \\
0 \\
L k_{f} s_{4}-k_{m} c_{4}
\end{array}\right]}
\end{gathered}
$$

the $3 \times 4$ input coupling matrixes $\left(s_{i}=\sin \left(\alpha_{i}\right)\right.$ and $c_{i}=$ $\left.\cos \left(\alpha_{i}\right)\right)$. Note that inputs $\boldsymbol{w}$ appear linearly in (7), as expected. As in many output tracking problems, a convenient solution is to resort to output feedback linearization techniques (either static or dynamic), see [18] for a detailed treatment. To this end, we rewrite the first two rows of (7) as

$$
\begin{aligned}
{\left[\begin{array}{c}
\ddot{\boldsymbol{p}} \\
\dot{\boldsymbol{\omega}}_{B}
\end{array}\right] } & \left.=\left[\begin{array}{c}
0 \\
0 \\
-g
\end{array}\right]\right]+\left[\begin{array}{cc}
\frac{1}{m}{ }^{W} \boldsymbol{R}_{B} & \mathbf{0} \\
\mathbf{0} & \boldsymbol{I}_{B}^{-1}
\end{array}\right]\left[\begin{array}{cc}
\boldsymbol{F}(\boldsymbol{\alpha}) & \mathbf{0} \\
\boldsymbol{\tau}(\boldsymbol{\alpha}) & \mathbf{0}
\end{array}\right]\left[\begin{array}{c}
\boldsymbol{w} \\
\boldsymbol{w}_{\alpha}
\end{array}\right] \\
& =\boldsymbol{f}+\boldsymbol{J}_{R}\left[\overline{\boldsymbol{J}}_{\alpha}(\boldsymbol{\alpha}) \quad \mathbf{0}\right]\left[\begin{array}{c}
\boldsymbol{w} \\
\boldsymbol{w}_{\alpha}
\end{array}\right]=\boldsymbol{f}+\boldsymbol{J}_{R} \boldsymbol{J}_{\alpha}(\boldsymbol{\alpha})\left[\begin{array}{c}
\boldsymbol{w} \\
\boldsymbol{w}_{\alpha}
\end{array}\right] \\
& =\boldsymbol{f}+\boldsymbol{J}(\boldsymbol{\alpha})\left[\begin{array}{c}
\boldsymbol{w} \\
\boldsymbol{w}_{\alpha}
\end{array}\right],
\end{aligned}
$$

where $\boldsymbol{f} \in \mathbb{R}^{6}$ is a constant drift vector, $\overline{\boldsymbol{J}}(\boldsymbol{\alpha}) \in \mathbb{R}^{6 \times 4}$, $\boldsymbol{J}_{R} \in \mathbb{R}^{6 \times 6}$, and the $6 \times 8$ matrix $\boldsymbol{J}(\boldsymbol{\alpha})$ will be referred to as the output Jacobian. Assuming the map (9) is invertible, that is $\rho_{J}=\operatorname{rank}(\boldsymbol{J}(\boldsymbol{\alpha}))=6$, it is always possible to statically feedback linearize (9) by means of the law

$$
\left[\begin{array}{c}
\boldsymbol{w} \\
\boldsymbol{w}_{\alpha}
\end{array}\right]=\boldsymbol{K}(\boldsymbol{\alpha})\left(-\boldsymbol{f}+\left[\begin{array}{c}
\ddot{\boldsymbol{p}}_{r} \\
\dot{\boldsymbol{\omega}}_{r}
\end{array}\right]\right)
$$

where $\boldsymbol{K}(\boldsymbol{\alpha})$ is a generalized inverse of $\boldsymbol{J}(\boldsymbol{\alpha})$, e.g., the pseudoinverse $\boldsymbol{J}^{\dagger}(\boldsymbol{\alpha})$, and $\left[\begin{array}{ll}\ddot{\boldsymbol{p}}_{r}^{T} & \dot{\boldsymbol{\omega}}_{r}^{T}\end{array}\right]^{T} \in \mathbb{R}^{6}$ an arbitrary reference linear/angular acceleration vector to be imposed to the output dynamics in (7).

However, this solution is not immediately viable in our case: in fact, $\rho_{J}=\operatorname{rank}(\boldsymbol{J})=\operatorname{rank}\left(\boldsymbol{J}_{R} \boldsymbol{J}_{\alpha}\right)=\operatorname{rank}\left(\boldsymbol{J}_{\alpha}\right)$ since $\boldsymbol{J}_{R}$ is a nonsingular square matrix. Furthermore, $\rho_{J}=$ $\operatorname{rank}\left(\boldsymbol{J}_{\alpha}\right)=\operatorname{rank}\left(\overline{\boldsymbol{J}}_{\alpha}\right) \leq 4<6$ because of the structural null matrix $\mathbf{0} \in \mathbb{R}^{6 \times 4}$ in matrix $\boldsymbol{J}_{\alpha}(\boldsymbol{\alpha})$ weighting the inputs $\boldsymbol{w}_{\alpha}$. Presence of this null matrix is due to the fact that inputs $\boldsymbol{w}_{\alpha}$ affect the output dynamics at a higher differential level compared to inputs $\boldsymbol{w}$. Therefore, a direct inversion at the acceleration level is bound to exploit only inputs $\boldsymbol{w}$ resulting in a loss of controllability for the system. Intuitively, the instantaneous linear/angular acceleration of the quadrotor body is directly affected by the propeller speeds $\boldsymbol{w}$ and tilting configuration $\boldsymbol{\alpha}$ (thanks to the dependance in $\overline{\boldsymbol{J}}_{\alpha}(\boldsymbol{\alpha})$ ), but not by the tilting velocities $\dot{\boldsymbol{\alpha}}=\boldsymbol{w}_{\alpha}$. It is interesting to note that this inhomogeneity in the differential levels at which inputs are affecting the output dynamics is not a specificity of the system at hand. As an example, the same structural property is also present in other robotic structures such as mobile manipulators with steering wheels [19] where the role of $\boldsymbol{w}_{\alpha}$ is played by the wheel steering velocities.

A possible way to circumvent these difficulties and regain controllability is to exploit the null space of $\boldsymbol{J}$ (of dimension $\geq 4$ ) in order to optimize some 'task feasibility' measure which, by acting on inputs $\boldsymbol{w}_{\alpha}$, will orient the $\rho_{J^{-}}$ dimensional range space of $\boldsymbol{J}(\boldsymbol{\alpha})\left(\rho_{J} \leq 4\right)$ so as to span the desired 6-dimensional (task) vector $\left(-\boldsymbol{f}+\left[\begin{array}{ll}\ddot{\boldsymbol{p}}_{r}^{T} & \dot{\boldsymbol{\omega}}_{r}^{T}\end{array}\right]^{T}\right)$ in (10). Alternatively, a somehow more systematic procedure is to resort to a dynamic output linearization scheme and seek to invert the input-output map at a higher differential level where inputs $\boldsymbol{w}_{\alpha}$ will explicitly appear. Throughout the rest of the Section we will focus on this latter approach.

By expanding the term $\overline{\boldsymbol{J}}_{\alpha}(\boldsymbol{\alpha}) \boldsymbol{w}$ in (9) as

$$
\overline{\boldsymbol{J}}_{\alpha}(\boldsymbol{\alpha}) \boldsymbol{w}=\sum_{i=1}^{4} \overline{\boldsymbol{j}}_{i}(\boldsymbol{\alpha}) w_{i}
$$

and noting that

$$
\frac{\mathrm{d} \overline{\boldsymbol{J}}_{\alpha}(\boldsymbol{\alpha}) \boldsymbol{w}}{\mathrm{d} t}=\overline{\boldsymbol{J}}_{\alpha}(\boldsymbol{\alpha}) \dot{\boldsymbol{w}}+\sum_{i=1}^{4} \frac{\partial \overline{\boldsymbol{j}}_{i}(\boldsymbol{\alpha})}{\partial \boldsymbol{\alpha}} \boldsymbol{w}_{\alpha} w_{i}
$$

differentiation of (9) w.r.t. time yields

$$
\begin{aligned}
& {\left[\begin{array}{c}
\dddot{\boldsymbol{p}} \\
\ddot{\boldsymbol{\omega}}_{B}
\end{array}\right]=\boldsymbol{J}_{R} \overline{\boldsymbol{J}}_{\alpha}(\boldsymbol{\alpha}) \dot{\boldsymbol{w}}+\boldsymbol{J}_{R} \sum_{i=1}^{4} \frac{\partial \overline{\boldsymbol{j}}_{i}(\boldsymbol{\alpha})}{\partial \boldsymbol{\alpha}} \boldsymbol{w}_{\alpha} w_{i}+\dot{\boldsymbol{J}}_{R} \overline{\boldsymbol{J}}_{\alpha}(\boldsymbol{\alpha}) \boldsymbol{w}}
\end{aligned}
$$

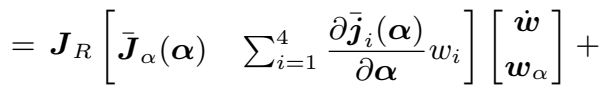

$$
\begin{aligned}
& {\left[\begin{array}{c}
\frac{{ }^{W} \dot{\boldsymbol{R}}_{B}}{m} \boldsymbol{F}(\boldsymbol{\alpha}) \boldsymbol{w} \\
\mathbf{0}
\end{array}\right]} \\
& =\boldsymbol{J}_{R} \boldsymbol{J}_{\alpha}^{\prime}(\boldsymbol{\alpha}, \boldsymbol{w})\left[\begin{array}{c}
\dot{\boldsymbol{w}} \\
\boldsymbol{w}_{\alpha}
\end{array}\right]+\boldsymbol{b}\left(\boldsymbol{\alpha}, \boldsymbol{w}, \boldsymbol{\omega}_{B}\right) \\
& =\boldsymbol{A}(\boldsymbol{\alpha}, \boldsymbol{w})\left[\begin{array}{c}
\dot{\boldsymbol{w}} \\
\boldsymbol{w}_{\alpha}
\end{array}\right]+\boldsymbol{b}\left(\boldsymbol{\alpha}, \boldsymbol{w}, \boldsymbol{\omega}_{B}\right)
\end{aligned}
$$

where the new input $\dot{\boldsymbol{w}}$ is the dynamic extension of the former (and actual) input $\boldsymbol{w}$ obtained by adding 4 integrators on its channel ${ }^{5}$. Note that the new $6 \times 8$ input-output decoupling matrix $\boldsymbol{A}(\boldsymbol{\alpha}, \boldsymbol{w})$ is made of two column blocks: while the first block $\boldsymbol{J}_{R} \overline{\boldsymbol{J}}_{\alpha}(\boldsymbol{\alpha})$ is exactly the first block of the

\footnotetext{
${ }^{5} \mathrm{By}$ means of this dynamic extensions, vector $\boldsymbol{w}$ becomes an internal state of the controller.
} 
former Jacobian $\boldsymbol{J}(\boldsymbol{\alpha})$, the second block is not a null matrix as in the previous case. Rather, a new set of 4 columns, weighting inputs $\boldsymbol{w}_{\alpha}$, are now present and contributing to the rank of matrix $\boldsymbol{A}$. It is particularly worth noting that $\boldsymbol{A}(\boldsymbol{\alpha}, \mathbf{0})=\boldsymbol{J}(\boldsymbol{\alpha})$ : when the propellers are not spinning $(\boldsymbol{w}=\mathbf{0})$, matrix $\boldsymbol{A}$ collapses back into the previous Jacobian matrix $\boldsymbol{J}$. Therefore, it will be mandatory for the controller to ensure $\boldsymbol{w} \neq \mathbf{0}$ at all times in order to keep the second block of $\boldsymbol{A}$ away from vanishing.

Assuming for now that matrix $\boldsymbol{A}$ has full row rank $\rho_{A}=$ $\operatorname{rank}(\boldsymbol{A})=6$, it is then possible to invert (11) by means of the law

$$
\left[\begin{array}{c}
\dot{\boldsymbol{w}} \\
\boldsymbol{w}_{\alpha}
\end{array}\right]=\boldsymbol{A}^{\dagger}\left(\left[\begin{array}{c}
\dddot{\boldsymbol{p}}_{r} \\
\ddot{\boldsymbol{\omega}}_{R}
\end{array}\right]-\boldsymbol{b}\right)+\left(\mathbf{I}_{8}-\boldsymbol{A}^{\dagger} \boldsymbol{A}\right) \boldsymbol{z},
$$

with $\mathbf{I}_{N}$ being the identity matrix of dimension $N$, in order to achieve full input-output linearization

$$
\left[\begin{array}{c}
\dddot{p}^{\prime} \\
\ddot{\omega}_{B}
\end{array}\right]=\left[\begin{array}{c}
\dddot{\boldsymbol{p}}_{r} \\
\ddot{\omega}_{R}
\end{array}\right] .
$$

The vector $\boldsymbol{z} \in \mathbb{R}^{8}$ in (14) is an additional free quantity projected onto the (2-dimensional) null space of $\boldsymbol{A}$ whose use will be detailed later on. We are now in position for solving the control problem stated at the beginning of the Section, i.e., asymptotic tracking of a desired trajectory $\left(\boldsymbol{p}_{d}(t), \boldsymbol{R}_{d}(t)\right)$. Assuming $\boldsymbol{p}_{d}(t) \in \overline{\mathcal{C}}^{3}$, it is sufficient to set

$$
\dddot{\boldsymbol{p}}_{r}=\dddot{\boldsymbol{p}}_{d}+\boldsymbol{K}_{p_{1}}\left(\ddot{\boldsymbol{p}}_{d}-\ddot{\boldsymbol{p}}\right)+\boldsymbol{K}_{p_{2}}\left(\dot{\boldsymbol{p}}_{d}-\dot{\boldsymbol{p}}\right)+\boldsymbol{K}_{p_{3}}\left(\boldsymbol{p}_{d}-\boldsymbol{p}\right)
$$

for obtaining exponential and decoupled convergence of the position error to $\mathbf{0}$ as long as the (diagonal) positive definite gain matrixes $\boldsymbol{K}_{p_{1}}, \boldsymbol{K}_{p_{2}}, \boldsymbol{K}_{p_{3}}$ define Hurwitz polynomials.

As for the stabilization of the orientation tracking error, several choices are possible depending on the particular parameterization chosen for the rotation matrix ${ }^{W} \boldsymbol{R}_{B}$. Besides the usual Euler angles (with their inherent singularity issues), an interesting possibility is to resort to an orientation error term directly defined on $S O(3)$, as shown in [20], [17]. Assume, as before, that $\boldsymbol{R}_{d}(t) \in \overline{\mathcal{C}}^{3}$ and let $\boldsymbol{\omega}_{d}=\left[\boldsymbol{R}_{d}^{T} \dot{\boldsymbol{R}}_{d}\right]_{\vee}$, where $[\cdot]_{\vee}$ represents the inverse map from $s o(3)$ to $\mathbb{R}^{3}$. By defining the orientation error as

$$
\boldsymbol{e}_{R}=\frac{1}{2}\left[{ }^{W} \boldsymbol{R}_{B}^{T} \boldsymbol{R}_{d}-\boldsymbol{R}_{d}^{T W} \boldsymbol{R}_{B}\right] \vee
$$

the choice

$$
\ddot{\boldsymbol{\omega}}_{r}=\ddot{\boldsymbol{\omega}}_{d}+\boldsymbol{K}_{\omega_{1}}\left(\dot{\boldsymbol{\omega}}_{d}-\dot{\boldsymbol{\omega}}_{B}\right)+\boldsymbol{K}_{\omega_{2}}\left(\boldsymbol{\omega}_{d}-\boldsymbol{\omega}_{B}\right)+\boldsymbol{K}_{\omega_{3}} \boldsymbol{e}_{R}
$$

yields an exponential convergence for the orientation tracking error to $\mathbf{0}$ as desired, provided that the (diagonal) gain matrixes $\boldsymbol{K}_{\omega_{1}}, \boldsymbol{K}_{\omega_{2}}, \boldsymbol{K}_{\omega_{3}}$ define a Hurwitz polynomial.

Note that, although the body linear/angular accelerations $\ddot{\boldsymbol{p}}$ and $\dot{\boldsymbol{\omega}}_{B}$ appear as feedback terms in (15)-(17), a direct, and possibly noisy, acceleration measurement is not strictly needed. In fact, these quantities can also be evaluated in terms of sole velocity measurements (vector $\boldsymbol{w}$ ) since, from (9), it follows that

$$
\left[\begin{array}{c}
\ddot{\boldsymbol{p}} \\
\dot{\boldsymbol{\omega}}_{B}
\end{array}\right]=\boldsymbol{f}+\boldsymbol{J}_{R} \overline{\boldsymbol{J}}_{\alpha}(\boldsymbol{\alpha}) \boldsymbol{w} .
$$

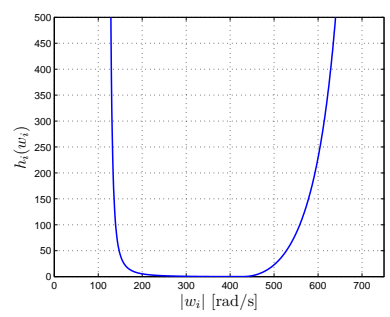

Fig. 4: An illustrative example of the functions $h_{i}\left(w_{i}\right)$ with $w_{\min }=$ $120[\mathrm{rad} / \mathrm{s}], w_{\text {rest }}=350[\mathrm{rad} / \mathrm{s}]$ and $w_{\max }=650[\mathrm{rad} / \mathrm{s}]$. Note how $h_{i}\left(w_{i}\right) \rightarrow \infty$ whenever $\left|w_{i}\right| \rightarrow w_{\min }$ or $\left|w_{i}\right| \rightarrow w_{\max }$, and has a minimum at $w_{\text {rest }}$ with continuous derivative

As a final step, we now discuss how to exploit the additional command $\boldsymbol{z}$ in (13). Being projected onto the null space of $\boldsymbol{A}$, vector $\boldsymbol{z}$ does not produce actions interfering with the output tracking objective and can thus be exploited to fulfill additional tasks. In our case, a first mandatory requirement is to keep $\rho_{A}=6$ at all times to avoid singularities of the decoupling matrix $\boldsymbol{A}$ in (13). As a matter of fact, matrix $\boldsymbol{A}$ has always rank $\rho_{A}=6$ as long as $w_{i} \neq 0$, $i=1 \ldots 4$, i.e., as long as the propellers are not stopping their spinning motion around $\boldsymbol{Z}_{P_{i}}$. While the necessity of such condition is an obvious consequence of the fact that, as explained before, $\boldsymbol{A}(\boldsymbol{\alpha}, \mathbf{0})=\boldsymbol{J}(\boldsymbol{\alpha})$, proof of its sufficiency will be given the Appendix ${ }^{6}$.

On the other hand, one would also like to minimize the norm of $\boldsymbol{w}$ in order to reduce the energy consumption during flight since, for instance, the air drag torques $\boldsymbol{\tau}_{\text {ext }}$ in (2) are always performing a dissipative work against $w_{i}$. A possibility in this sense is to keep each $w_{i}$ below a maximum propeller speed $w_{\max }$. As possible cost function $H(\boldsymbol{w})$ taking into account these two competing objectives, we propose $H(\boldsymbol{w})=\sum_{i=1}^{4} h\left(w_{i}\right)$ where $h\left(w_{i}\right)=$

$$
\left\{\begin{array}{cc}
k_{h_{1}} \tan ^{2}\left(\gamma_{1}\left|w_{i}\right|+\gamma_{2}\right) & w_{\text {min }}<\left|w_{i}\right| \leq w_{\text {rest }} \\
k_{h_{2}}\left(\left|w_{i}\right|-w_{\text {rest }}\right)^{2}-\frac{\left(\left|w_{i}\right|-w_{\text {rest }}\right)^{2}}{\left(\left|w_{i}\right|-w_{\text {max }}\right)} & \left|w_{i}\right|>w_{\text {rest }}
\end{array}\right.
$$

with $\gamma_{1}=\frac{\pi}{2\left(w_{\text {rest }}-w_{\text {min }}\right)}, \gamma_{2}=-\gamma_{1} w_{\text {rest }}$, and $k_{h_{1}}>0$, $k_{h_{2}}>0$ suitable scalar gains. Here, $w_{\min }>0$ represents a minimum value for the propeller spinning velocities, $w_{\text {rest }}>$ $w_{\min }$ a suitable 'rest' speed, $w_{\max }>w_{\text {rest }}$ a maximum propeller speed, and the single $h_{i}\left(w_{i}\right)$ functions are such that $h_{i}\left(w_{i}\right) \rightarrow \infty$ if either $\left|w_{i}\right| \rightarrow w_{\min }$ or $\left|w_{i}\right| \rightarrow w_{\max }$, and have a minimum (with continuous derivative) in $w_{\text {rest }}$. An illustrative example for $h_{i}\left(w_{i}\right)$ with $w_{\min }=120[\mathrm{rad} / \mathrm{s}]$, $w_{\text {rest }}=350[\mathrm{rad} / \mathrm{s}]$ and $w_{\max }=650[\mathrm{rad} / \mathrm{s}]$ is shown in Fig. 4. This values correspond to our quadrotor motor propeller combination. Minimization of $H(\boldsymbol{w})$, compatibly with the output tracking task, is then obtained by setting in (13)

$$
\boldsymbol{z}=-k_{H}\left[\begin{array}{c}
\nabla_{\boldsymbol{w}} H(\boldsymbol{w}) \\
\mathbf{0}
\end{array}\right]
$$

\footnotetext{
${ }^{6}$ It is interesting to note that this property is, again, conceptually equivalent to the case of a nonholonomic mobile manipulator with steering wheels where a nonzero spinning velocity of one wheel is required to guarantee non singularity of most task Jacobians, see [19] for more details.
} 


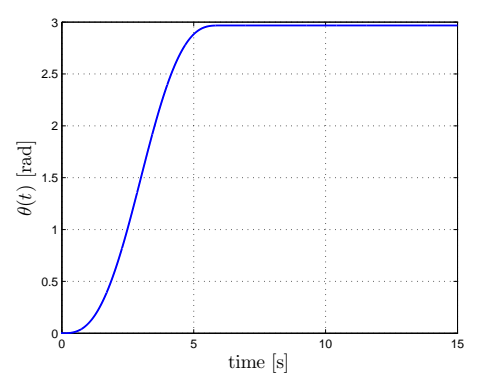

Fig. 5: Profile of $\theta(t)$ for the first simulation

with $k_{H}>0$ being a suitable stepsize. Note that, as a byproduct, this choice will also result in a beneficial 'velocity damping'-like action on the states $\boldsymbol{w}$ as, e.g., described in [21].

\section{SIMULATIONS}

In the following, we will present two simulations performed by applying the controller presented in the previous Section on the dynamic model described in Sect. II. The aim is twofold: on one side, we want to highlight the tracking capabilities of the proposed controller and the beneficial action of the null-space term (19) in avoiding singularities for matrix $\boldsymbol{A}$. On the other side, we also want to test the robustness of the controller against all the inertial/gyroscopic effects neglected at the control design stage but included in the quadrotor dynamic model (1)-(3)-(6).

\section{A. First simulation}

In this first simulation, we tested a simple trajectory involving a rotation of $0.94 \pi[\mathrm{rad}]$ on the spot along the $\boldsymbol{X}_{B}$ axis (this motion would be clearly unfeasible for a standard quadrotor). The initial conditions were set to $\boldsymbol{p}\left(t_{0}\right)=\mathbf{0}$, $\dot{\boldsymbol{p}}\left(t_{0}\right)=\mathbf{0},{ }^{W} \boldsymbol{R}_{B}\left(t_{0}\right)=\mathbf{I}_{3}, \boldsymbol{\omega}_{B}\left(t_{0}\right)=\mathbf{0}, \boldsymbol{\alpha}\left(t_{0}\right)=\mathbf{0}$, $\dot{\boldsymbol{\alpha}}\left(t_{0}\right)=\mathbf{0}$, and $\boldsymbol{w}\left(t_{0}\right)=w_{\text {rest }}$. The desired trajectory was chosen as $\boldsymbol{p}_{d}(t) \equiv 0$ and $\boldsymbol{R}_{d}(t)=\boldsymbol{R}_{X}(\theta(t))$ with $\theta(t)$ following the smooth profile shown in Fig. 5 with maximum velocity $\dot{\theta}_{\max }=1[\mathrm{rad} / \mathrm{s}]$ and maximum acceleration $\ddot{\theta}_{\max }=0.5\left[\mathrm{rad} / \mathrm{s}^{2}\right]$. The trajectory was executed twice by $(i)$ including and $(i i)$ not including the null-space term $\boldsymbol{z}$ (19) into (13) $\left(k_{H}=1\right.$ or $\left.k_{H}=0\right)$. The gains in (15)-(17) were set to $\boldsymbol{K}_{p_{1}}=\boldsymbol{K}_{\omega_{1}}=7.5 \mathbf{I}_{3}, \boldsymbol{K}_{p_{2}}=\boldsymbol{K}_{\omega_{2}}=18.75 \mathbf{I}_{3}$, $\boldsymbol{K}_{p_{3}}=\boldsymbol{K}_{\omega_{3}}=15.62 \mathbf{I}_{3}$, and $k_{h_{1}}=1, k_{h_{2}}=10$ in (18).

Figures $6(\mathrm{a}-\mathrm{f})$ show the results of the simulation in these two cases. In particular, Fig. 6(a) shows the superimposition of $H(\boldsymbol{w})$ when including $\boldsymbol{z}$ (red dashed line, case $(i)$ ) and not including $\boldsymbol{z}$ (blue solid line, case $(i i)$ ). It is clear that, in the latter case, $H(\boldsymbol{w})$ attains a lower value over time thanks to the optimization action in (19). As a consequence, this results in a lower value for $\|\boldsymbol{w}\|$ over time as depicted in Fig. 6(b) (same color pattern), showing that the given task (rotation on the spot) can be realized in a more 'energy-efficient' way when properly shaping the cost function $H(\boldsymbol{w})$. Note that, as a byproduct, the better performance of case $(i)$ comes at the expense of a more complex reorientation of the propeller groups during the motion. This is shown in Figs. 6(c-d) which report the behavior of the 4 tilt angles

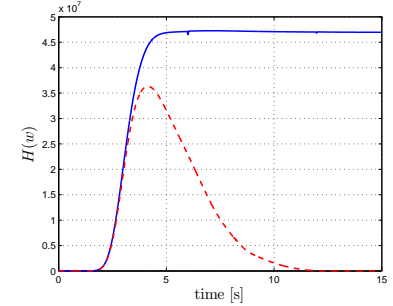

(a)

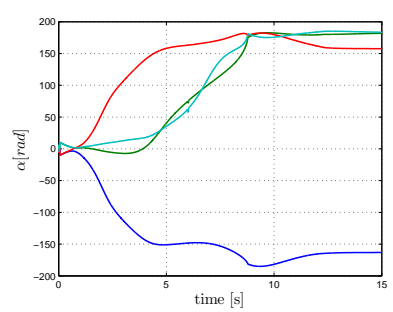

(c)

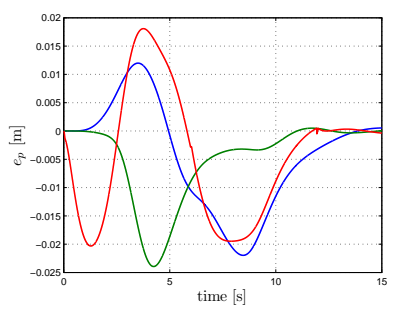

(e)

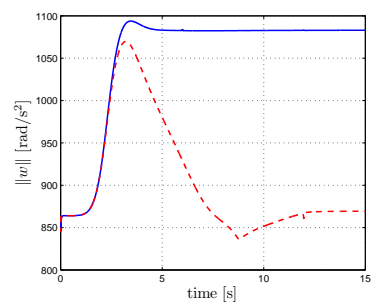

(b)

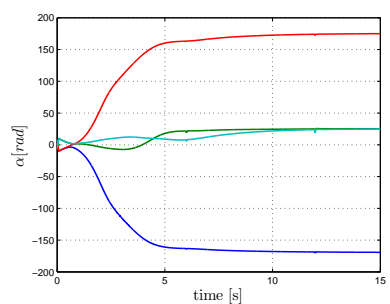

(d)

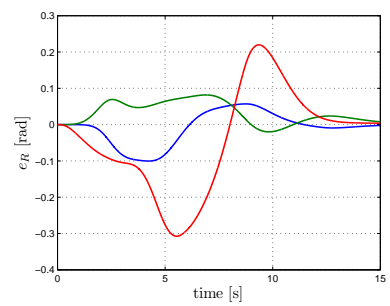

(f)
Fig. 6: Results of the first simulation with $(i)$ and without $(i i)$ exploiting the null-space term (19). 6(a): behavior of $H(\boldsymbol{w})$ for cases $(i)$ (red dashed line) and (ii) (blue solid line). 6(b): behavior of $\|w\|$ for cases $(i)$ (red dashed line) and (ii) (blue solid line). 6(c-d): behavior of the tilt angles $\boldsymbol{\alpha}$ for cases (i) (left) and (ii) (right). 6(e-f): behavior of the position/orientation tracking errors $\left(\boldsymbol{e}_{p}, \boldsymbol{e}_{R}\right)$ for case $(i i)$.

$\alpha_{i}$ in cases $(i)$ (left) and ( $\left.i i\right)$ (right): compared to Fig. 6(d), note the rotation of two propellers starting from $t \approx 4[s]$ in Fig. 6(c). Finally, Figs. 6(e-f) show the position tracking error $\boldsymbol{e}_{p}(t)=\boldsymbol{p}_{d}(t)-\boldsymbol{p}(t)$ and orientation tracking error $\boldsymbol{e}_{R}(t)$ (16) only for case $(i)$. Despite the fast reorientation of two propellers highlighted in Fig. 6(d), the tracking errors stay small (note the scales) and eventually converge to zero as the desired trajectory comes to a full stop. This provides a first confirmation of the validity of our assumptions in Sect. III, that is, robustness of the controller w.r.t. any gyroscopic/inertial effect due to the internal relative motion of the different bodies composing the quadrotor. This point will also be addressed more thoroughly by the next simulation.

For the reader's convenience, we also report in Figs. 7(ab) a series of snapshots illustrating the quadrotor motion in these two cases.

\section{B. Second simulation}

In the second simulation, we addressed the case of a more complex trajectory following a square path with vertexes $\left\{V_{1}, V_{2}, V_{3}, V_{4}, V_{5}\right\}$. Each vertex was associated with the 

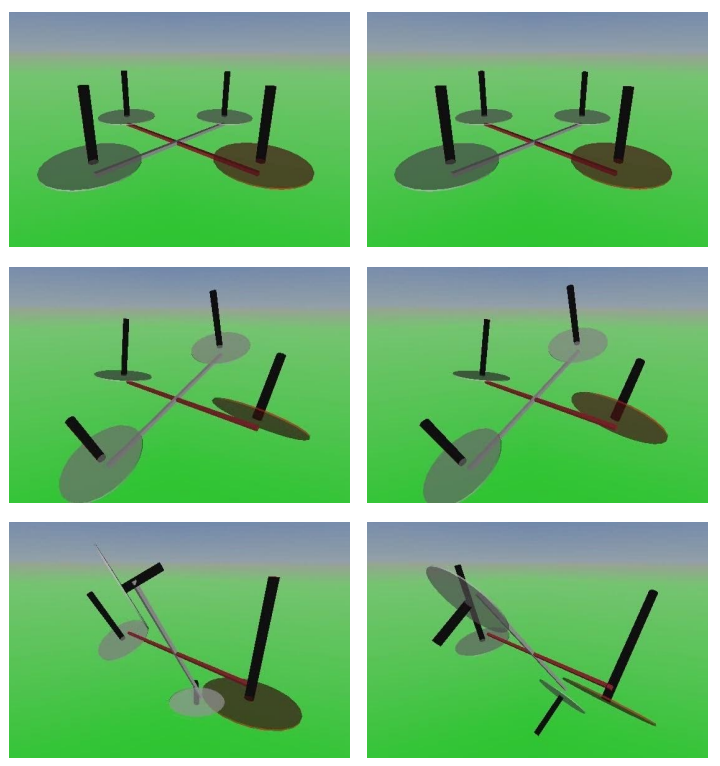

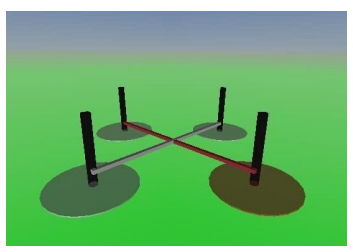

(a)

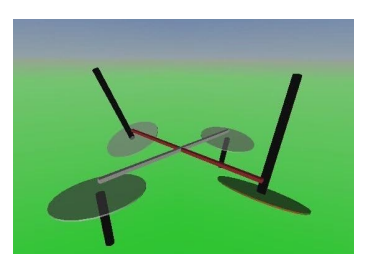

(b)
Fig. 7: Results of the first simulation. Left column: quadrotor motion during case $(i)$. Right column: quadrotor motion during case $(i i)$. Note the large reorientation of the propeller groups in this latter case thanks to the action of the optimization term (19)

following desired positions and orientations ${ }^{7}$

- $\boldsymbol{V}_{1}: \boldsymbol{p}_{d}=\left[\begin{array}{lll}0 & 0 & 0\end{array}\right]^{T}, \boldsymbol{\eta}_{d}=\left[\begin{array}{lll}0 & 0 & 0\end{array}\right]^{T}$

- $\boldsymbol{V}_{2}: \boldsymbol{p}_{d}=\left[\begin{array}{lll}0 & 3 & 1\end{array}\right]^{T}, \boldsymbol{\eta}_{d}=\left[\begin{array}{lll}0 & -\pi / 2 & 0\end{array}\right]^{T}$

- $\boldsymbol{V}_{3}: \boldsymbol{p}_{d}=\left[\begin{array}{lll}2 & 3 & 1\end{array}\right]^{T}, \boldsymbol{\eta}_{d}=\left[\begin{array}{lll}-\pi / 4 & 0 & -\pi / 4\end{array}\right]^{T}$

- $\boldsymbol{V}_{4}: \boldsymbol{p}_{d}=\left[\begin{array}{lll}2 & 0 & 0\end{array}\right]^{T}, \boldsymbol{\eta}_{d}=\left[\begin{array}{lll}-1.41 & -1.08 & 0.25\end{array}\right]^{T}$

- $\boldsymbol{V}_{5}: \boldsymbol{p}_{d}=\left[\begin{array}{lll}0 & 0 & 0\end{array}\right]^{T}, \boldsymbol{\eta}_{d}=\left[\begin{array}{lll}0.31 & -1.08 & 0.25\end{array}\right]^{T}$

which were traveled along with rest-to-rest motions with maximum linear/angular velocities of $1[\mathrm{~m} / \mathrm{s}]$ and $1[\mathrm{rad} / \mathrm{s}]$, and maximum linear/angular accelerations of $1\left[\mathrm{~m} / \mathrm{s}^{2}\right]$ and $0.2\left[\mathrm{rad} / \mathrm{s}^{2}\right]$. Figure 8 shows a series of snapshots illustrating the overall motion, while Figs. 9(a-d) show the desired trajectory $\left(\boldsymbol{p}_{d}(t), \boldsymbol{\eta}_{d}(t)\right)$, and the tracking errors $\left(\boldsymbol{e}_{p}(t), \boldsymbol{e}_{R}(t)\right)$. Note again how the tracking errors keep limited as before despite the more complex motion involving several reorientations of the propellers. This confirms again the validity of the controller proposed in the previous Section. The interested reader can also refer to the video attached to the paper for a more exhaustive illustration of the quadrotor motion capabilities.

\section{CONCLUSIONS AND FUTURE WORKS}

In this paper, we have addressed the modeling and control issues for a quadrotor UAV with a novel actuation concept which allows the 4 propellers to actively rotate (tilt) about the axes connecting them to the quadrotor main body. The

\footnotetext{
${ }^{7}$ Here, for the sake of clarity, we represent orientations by means of the classical roll/pitch/yaw Euler set $\boldsymbol{\eta} \in \mathbb{R}^{3}$.
}

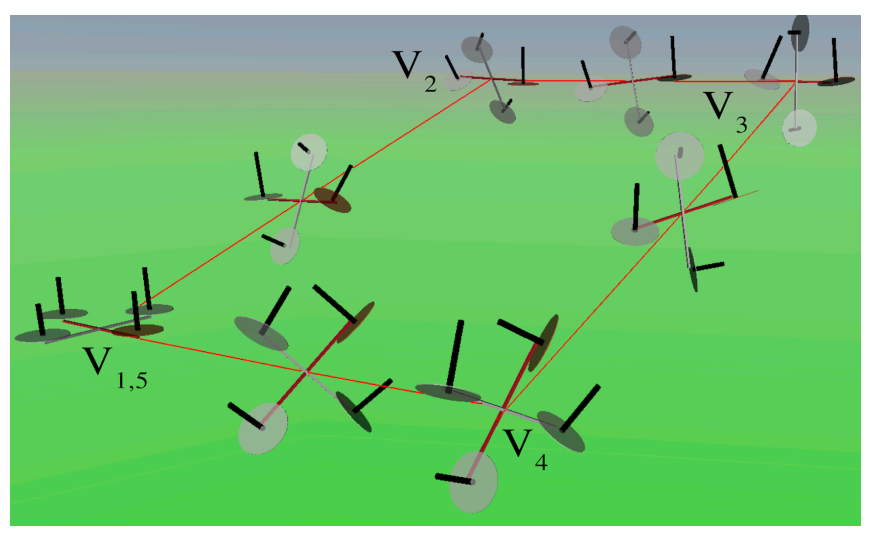

Fig. 8: Trajectory (red line) with SQC visualized in according orientations during the maneuver

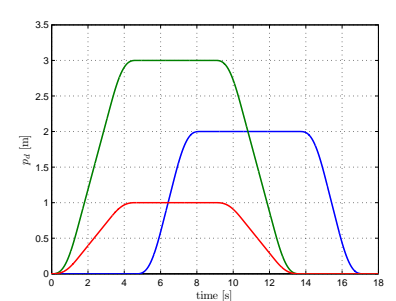

(a)

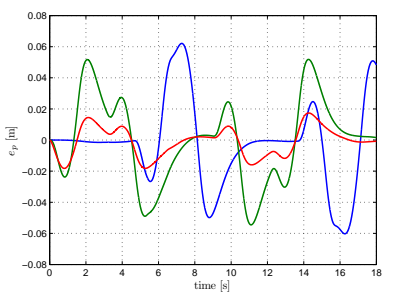

(c)

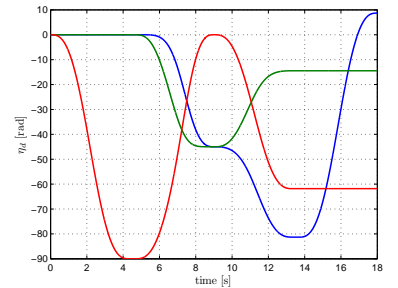

(b)

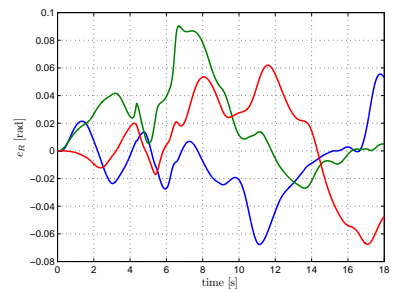

(d)
Fig. 9: Fig. 9-a: Desired position $p_{d}$ in $\mathrm{x}, \mathrm{y}$ and z; Fig. 9-b: Desired orientation $\eta$ in roll, pitch and yaw; Fig. 9-c: Position tracking error $e_{p}$; Fig. 9-c: Orientation tracking error $e_{R}$;

addition of this new set of 4 control inputs makes it possible to $(i)$ gain full controllability over the 6 -dof quadrotor pose, and (ii) exploit the resulting actuation redundancy (of order 2) so as to optimize suitable energy-efficiency criteria. After carefully analyzing the controllability properties of the system, we proposed a trajectory tracking controller based on dynamic feedback linearization techniques, and provided a validation by means of extensive simulations on a number of reference trajectories.

Our next steps are aimed at, on one side, devising and comparing additional control designs based on different techniques (e.g., backstepping, sliding mode), and, on the other side, at realizing a first prototype of the proposed quadrotor in order to work towards an actual implementation of the ideas discussed in this paper.

\section{APPENDIX}

Propositon 1: If $w_{i} \neq 0, i=1 \ldots 4$, then $\rho_{A}=$ $\operatorname{rank}(\boldsymbol{A})=6$.

Proof: From (11), note that $\operatorname{rank}(\boldsymbol{A})=$ $\operatorname{rank}\left(\boldsymbol{J}_{R} \boldsymbol{J}_{\alpha}^{\prime}\right)=\operatorname{rank}\left(\boldsymbol{J}_{\alpha}^{\prime}\right)$ since $\boldsymbol{J}_{R}$ is a square nonsingular 
matrix. We now look for suitable nonsingular matrixes $\boldsymbol{M} \in \mathbb{R}^{6 \times 6}$ and $\boldsymbol{N} \in \mathbb{R}^{8 \times 8}$ such that $\boldsymbol{M} \boldsymbol{J}_{\alpha}^{\prime} \boldsymbol{N}$ is in row canonical form, that is

$$
\boldsymbol{M} \boldsymbol{J}_{\alpha}^{\prime} \boldsymbol{N}=\left[\mathbf{I}_{6} \mid *\right] .
$$

If this manipulation is possible, then $\operatorname{rank}(\boldsymbol{A})=$ $\operatorname{rank}\left(\boldsymbol{J}_{\alpha}^{\prime}\right)=\operatorname{rank}\left(\boldsymbol{M} \boldsymbol{J}_{\alpha}^{\prime} \boldsymbol{N}\right)=6$. By inspection of $\boldsymbol{J}_{\alpha}^{\prime}$, and after some (tedious) manipulations, a suitable $M$ can be found as

$$
\boldsymbol{M}=\left[\begin{array}{cccccc}
M_{11} & M_{12} & M_{13} & M_{14} & M_{15} & M_{16} \\
M_{21} & 0 & 0 & M_{24} & 0 & 0 \\
M_{31} & M_{32} & M_{33} & M_{34} & M_{35} & M_{36} \\
M_{41} & M_{42} & M_{43} & M_{44} & M_{45} & M_{46} \\
M_{51} & 0 & 0 & M_{54} & 0 & 0 \\
M_{61} & M_{62} & M_{63} & M_{64} & M_{65} & M_{66}
\end{array}\right]
$$

with

$$
\left\{\begin{array}{cc}
M_{11}=-\frac{k_{m} k_{f} L s_{1}+\left(2 k_{m}^{2}+k_{f}^{2} L^{2}\right) c_{1}}{2 L^{2} k_{f}^{3} w_{1}}, & M_{12}=-\frac{L k_{f} c_{1}-k_{m} s_{1}}{2 L k_{f}^{2} w_{1}}, \\
M_{13}=-\frac{L k_{f} s_{1}+k_{m} c_{1}}{2 L k_{f}^{2} w_{1}}, & M_{14}=\frac{L k_{f} s_{1}+2 k_{m} c_{1}}{2 L^{2} k_{f}^{2} w_{1}}, \\
M_{15}=\frac{s_{1}}{2 L k_{f} w_{1}}, & M_{16}=-\frac{c_{1}}{2 L k_{f} w_{1}}, \\
M_{21}=-\frac{L k_{f} c_{2}+k_{m} s_{2}}{L k_{f}^{2} w_{2}}, & M_{24}=\frac{s_{2}}{L k_{f} w_{2}}, \\
M_{31}=-\frac{-L k_{m} k_{f} c_{3}+\left(2 k_{m}^{2}+L^{2} k_{f}^{2}\right) s_{3}}{2 L^{2} k_{f}^{3}}, & M_{32}=\frac{L k_{f} s_{3}+k_{m} c_{3}}{2 L k_{f}^{2}}, \\
M_{33}=\frac{L k_{f} c_{3}-k_{m} s_{3}}{2 L k_{f}^{2}}, & M_{34}=-\frac{L k_{f} c_{3}-2 k_{m} s_{3}}{2 L^{2} k_{f}^{2}}, \\
M_{35}=\frac{c_{3}}{2 L k_{f}}, & M_{36}=-\frac{s_{3}}{2 L k_{f}}, \\
M_{41}=-\frac{-L k_{m} k_{f} c_{1}+\left(2 k_{m}^{2}+L^{2} k_{f}^{2}\right) s_{1}}{2 L^{2} k_{f}^{3}}, & M_{42}=-\frac{L k_{f} s_{1}+k_{m} c_{1}}{2 L k_{f}^{2}}, \\
M_{43}=\frac{L k_{f} c_{1}-k_{m} s_{1}}{2 L k_{f}^{2}}, & M_{44}=-\frac{L k_{f} c_{1}-2 k_{m} s_{1}}{2 L^{2} k_{f}^{2}}, \\
M_{45}=-\frac{c_{1}}{2 L k_{f}}, & M_{46}=-\frac{s_{1}}{2 L k_{f}}, \\
M_{51}=-\frac{L k_{f} s_{2}-k_{m} c_{2}}{L k_{f}^{2}}, & M_{54}=-\frac{c_{2}}{L k_{f}}, \\
M_{61}=-\frac{L k_{f} k_{m} s_{3}+\left(2 k_{m}^{2}+L^{2} k_{f}^{2}\right) c_{3}}{2 L^{2} k_{f}^{3} w_{3}}, & M_{62}=\frac{L k_{f} c_{3}-k_{m} s_{3}}{2 L k_{f}^{2} w_{3}}, \\
M_{63}=-\frac{L k_{f} s_{3}+k_{m} c_{3}}{2 L k_{f}^{2} w_{3}}, & M_{64}=\frac{L k_{f} s_{3}+2 k_{m} c_{3}}{2 L^{2} k_{f}^{2} w_{3}}, \\
M_{65}=-\frac{s_{3}}{2 L k_{f} w_{3}}, & M_{66}=-\frac{c_{1}}{2 L k_{f}^{2} w_{3}},
\end{array}\right.
$$

and $\operatorname{det}(\boldsymbol{M})=-\frac{1}{4 L^{3} k_{f}^{6} w_{1} w_{2} w_{3}}$. As for $\boldsymbol{N}$, it is just a column permutation matrix

$$
\boldsymbol{N}=\left[\begin{array}{llllllll}
1 & 0 & 0 & 0 & 0 & 0 & 0 & 0 \\
0 & 1 & 0 & 0 & 0 & 0 & 0 & 0 \\
0 & 0 & 1 & 0 & 0 & 0 & 0 & 0 \\
0 & 0 & 0 & 0 & 0 & 0 & 1 & 0 \\
0 & 0 & 0 & 1 & 0 & 0 & 0 & 0 \\
0 & 0 & 0 & 0 & 1 & 0 & 0 & 0 \\
0 & 0 & 0 & 0 & 0 & 1 & 0 & 0 \\
0 & 0 & 0 & 0 & 0 & 0 & 0 & 1
\end{array}\right]
$$

It is then easy to see that, if $w_{1} \neq 0, w_{2} \neq 0$ and $w_{3} \neq 0$, matrix $M$ is always well-defined and (20) always holds, proving that $\rho_{A}=\operatorname{rank}(\boldsymbol{A})=6$. Note that in this case, because of the particular choice made for $M$, no condition is present on $w_{4}$ ( $w_{4}$ could vanish). This is coherent with the intuition that three actuated propellers can be sufficient to provide full mobility to the quadrotor body (6 inputs for 6 rigid body dofs). However, presence of a fourth active propeller $\left(w_{4} \neq 0\right.$ in this case) guarantees the needed actuation redundancy exploited in Sect. III.

\section{ACKNOWLEDGEMENTS}

This research was partly supported by WCU (World Class University) program funded by the Ministry of Education, Science and Technology through the National Research Foundation of Korea (R31-10008).

\section{REFERENCES}

[1] D. Gurdan, J. STumpf, M. Achtelik, K.-M. Dorh, G. Hirzinger, and D. Rus, "Energy-efficient autonomous four-rotor flying robot controlled at $1 \mathrm{kHz}$," in 2007 IEEE Int. Conf. on Robotics and Automation, 2007, pp. 361-366.

[2] S. Bouabdallah, M. Becker, and R. Siegwart, "Autonomous miniature flying robots: Coming soon!" IEEE Robotics and Automation Magazine, vol. 13, no. 3, pp. 88-98, 2007.

[3] K. P. Valavanis, Ed., Advances in Unmanned Aerial Vehicles: State of the Art and the Road to Autonomy. Springer, 2007.

[4] D. Mellinger, N. Michael, and V. Kumar, "Trajectory generation and control for precise aggressive maneuvers with quadrotors," in Proc. of the 2010 Int. Symposium on Experimental Robotics, 2010.

[5] P. Pounds, R. Mahony, and P. Corke, "Modelling and control of a large quadrotor robot," Control Engineering Practice, vol. 18, no. 7, pp. 691-699, 2010.

[6] L. Gentili, R. Naldi, and L. Marconi, "Modelling and control of VTOL UAVs interacting with the environment," in 2008 IEEE Conf. on Decision and Control, 2008, pp. 1231-1236.

[7] R. Naldi and L. Marconi, "Modeling and control of the interaction between flying robots and the environment," in Proc. of the 2010 IFAC NOLCOS, 2010.

[8] P. E. I. Pound, D. R. Bersak, and A. M. Dollar, "Grasping from the air: Hovering capture and load stability," in 2011 IEEE Int. Conf. on Robotics and Automation, 2011, pp. 2491-2498.

[9] Q. Lindsey, D. Mellinger, and V. Kumar, "Construction of cubic structures with quadrotor teams," in Robotics: Science and Systems, 2011.

[10] EU Collaborative Project ICT-248669 AIRobots, "www.airobots.eu."

[11] R. Naldi, L. Gentili, L. Marconi, and A. Sala, "Design and experimental validation of a nonlinear control law for a ducted-fan miniature aerial vehicle," Control Engineering Practice, vol. 18, no. 7, pp. 747$760,2010$.

[12] K. T. Oner, E. Cetinsoy, M. Unel, M. F. Aksit, I. Kandemir, and K. Gulez, "Dynamic model and control of a new quadrotor unmanned aerial vehicle with tilt-wing mechanism," in Proc. of the 2008 World Academy of Science, Engineering and Technology, 2008, pp. 58-63.

[13] K. T. Oner, E. Cetinsoy, E. Sirimoglu, C. Hancer, T. Ayken, and M. Unel, "LQR and SMC stabilization of a new unmanned aerial vehicle," in Proc. of the 2009 World Academy of Science, Engineering and Technology, 2009, pp. 554-559.

[14] F. Kendoul, I. Fantoni, and R. Lozano, "Modeling and control of a small autonomous aircraft having two tilting rotors," IEEE Trans. on Robotics, vol. 22, no. 6, pp. 1297-1302, 2006.

[15] A. Sanchez, J. Escareño, O. Garcia, and R. Lozano, "Autonomous hovering of a noncyclic tiltrotor UAV: Modeling, control and implementation," in Proc. of the 17th IFAC Wold Congress, 2008, pp. 803808.

[16] V. Mistler, A. Benallegue, and N. K. M'Sirdi, "Exact linearization and noninteracting control of a 4 rotors helicopter via dynamic feedback," in Proc. of the 10th IEEE International Workshop on Robot and Human Interactive Communication, 2001, pp. 586-593.

[17] D. Mellinger and V. Kumar, "Minimum snap trajectory generation and control for quadrotors," in 2011 IEEE Int. Conf. on Robotics and Automation, 2011, pp. 2520-2525.

[18] A. Isidori, Nonlinear Control Systems, 3rd ed. Springer, 1995.

[19] A. De Luca, G. Oriolo, and P. Robuffo Giordano, "Kinematic control of nonholonomic mobile manipulators in the presence of steering wheels," in 2010 IEEE Int. Conf. on Robotics and Automation, 2010, pp. 1792-1798.

[20] T. Lee, M. Leok, and N. H. McClamroch, "Geometric tracking control of a quadrotor UAV on SE(3)," in 2010 IEEE Conf. on Decision and Control, 2010, pp. 5420-5425.

[21] A. De Luca, G. Oriolo, and B. Siciliano, "Robot redundancy resolution at the acceleration level," Robotica, vol. 4, no. 2, pp. 97-106, 1992. 